\title{
Análise do P\&D Brasileiro com Uso da Nanotecnologia em Medicamentos para Consumo Humano: pesquisas, tecnologias e produtos
}

\author{
Brazilian R\&D with Respect to Nanotechnology in Drug Development: \\ research, technology and products
}

\author{
Tiago Giuriatti ${ }^{1}$ \\ Manuel Adalberto Alfaro De Práa \\ Fernando Richartz 3 \\ Eduardo Luiz Gasnhar Moreira ${ }^{3}$ \\ ${ }^{1}$ Instituto Federal Catarinense, Blumenau, SC, Brasil \\ ${ }^{2}$ Entourage Phytolab, Valinhos, SP, Brasil \\ ${ }^{3}$ Universidade Federal de Santa Catarina, Florianópolis, SC, Brasil
}

\begin{abstract}
Resumo
Este trabalho teve como objetivo analisar a dinâmica de relações entre Instituições de Ciência e Tecnologia (ICTs) e empresas brasileiras acerca de pesquisas envolvendo nanotecnologia com aplicação ao desenvolvimento de medicamentos para uso em seres humanos. Especificamente, buscou-se apresentar o processo de inovação promovido pela nanotecnologia nas pesquisas de origem brasileira, por meio do levantamento de linhas de pesquisas, depósitos de patentes, tecnologias desenvolvidas, transferências desses conhecimentos, ensaios clínicos em andamento $e$ concluídos, os quais resultaram em produtos de origem nacional. Observou-se que as empresas brasileiras, em termos de depósitos de patentes, apresentam números incipientes, embora um número expressivo desses depósitos seja oriundo de parceria com ICTs brasileiras. Em especial, algumas empresas brasileiras realizaram ensaios clínicos com parcerias de ICTs, resultando no desenvolvimento de produtos de origem brasileira.
\end{abstract}

Palavras-chave: Nanotecnologia. Medicamentos. Patentes.

\begin{abstract}
The aim of the present work was to analyze the relation between Science and Technology Institutes and the national pharmaceutical companies with respect to nanotechnology in drug development process. Specifically, it was analyzed the innovative process promoted by nanotechnology in Brazil, by evaluating the scientific research lines, deposits of patents, technologies developed, ongoing and completed clinical trials and, eventually, products in the market. Overall, it was observed a significant increase in the number of ongoing research studies and deposits of patents from national origin; however, the main portion of these deposits is from National Science and Technology Institutes. Noteworthy, there are national industries that developed clinical trials with national academy collaboration, which resulted in the development of pharmaceutical products.
\end{abstract}

Keywords: Nanotechnology. Drugs. Patents.

Área Tecnológica: Nanotecnologia. 


\section{Introdução}

Dados obtidos pela Associação Brasileira de Desenvolvimento Industrial (ABDI, 2013) mostram que, embora os investimentos anuais em pesquisa e desenvolvimento (P\&D) por empresas farmacêuticas, em todo o mundo, aumentaram de cerca de US\$ 1 bilhão em 1975 para US\$ 40 bilhões até o ano de 2013, a aprovação de novos fármacos permaneceu estável, entre 20 e 30 por ano. Uma parte pode ser compreendida em função de maiores imposições das agências reguladoras, exigindo comprovação de segurança e eficácia; além da saturação de novos alvos biológicos (KAITIN, 2010).

Nesse sentido, Liu, Constantinides e Li (2014) propõem que o estabelecimento de novas estratégias ao desenvolvimento de medicamentos, em conjunto com a integração de vários processos inovadores, devem aumentar, significativamente, as atividades de P\&D. Ainda, de acordo com Kaitin (2010), está ocorrendo uma mudança gradual nas relações e alianças da indústria farmacêutica, com uma alteração no tradicional modelo de indústria totalmente integrada nas atividades de pesquisa, no qual esta é responsável por todos os processos no desenvolvimento dos medicamentos. Nesse contexto de interações e de mudanças de relações, o autor supracitado aponta que os centros de pesquisa acadêmica subsidiam a pesquisa básica, base de todo o processo de inovação farmacêutica. Segundo o mesmo autor, importantes laboratórios, como Pfizer, Novartis, Genzyme, Sanofi e Janssen estão reorganizando seus processos de P\&D de novos medicamentos, nos quais a maioria passa por alianças com universidades. Ademais, nota-se uma tendência na aquisição de pequenas empresas farmacêuticas (e.g., startups) por empresas de grande porte, ou mesmo parcerias de desenvolvimento conjunto.

A inovação no setor farmacêutico pode surgir a partir da modificação de uma ou mais propriedades do composto, incluindo a estrutura química ou o método de síntese do princípio ativo, a forma farmacêutica, características farmacodinâmicas ou farmacocinéticas, bem como outras propriedades terapêuticas (ARONSON, 2008). Nesse sentido, atualmente, observa-se uma tendência das indústrias farmacêuticas em empregar a nanotecnologia ao desenvolvimento de novas formulações, principalmente relacionadas à melhorias na absorção e biodisponibilidade dos compostos de interesse. De fato, as aplicações da nanotecnologia na área médica e, mais especificamente, em sistemas de liberação controlada de fármacos estão se consolidando rapidamente. $\mathrm{O}$ grande interesse no estudo desses materiais deve-se ao fato de apresentarem propriedades físico-químicas distintas dos materiais-base já conhecidos, o que permite inúmeras novas possibilidades de aplicações, entre elas nanomedicamentos e nanocarreadores para o uso terapêutico, adjuvantes terapêuticos, sistemas de diagnóstico, entre outros produtos para a saúde (FALLEIROS et al., 2011 apud CAIXETA; BINSFELD, 2013). O mercado de nanomedicina foi avaliado em US\$248,3 bilhões em 2014. Há uma previsão que o mercado total cresça a uma taxa anual de 16,3\%, entre 2014 a 2019, e alcance US\$ 528 bilhões até o ano de 2019 (EVERS, 2015). Assim, com o avanço na apropriação de conhecimento na área de nanotecnologia, as indústrias farmacêuticas desenvolveram novas estratégias e técnicas para "re-patentear" fármacos que estão a caminho do vencimento, modificando-os por meio de nanotecnologia (FAIYAZUDDIN, 2014).

No Brasil, o desenvolvimento da área de nanotecnologia vem sendo estimulada pelo governo desde o início da década de 2000. Pode-se citar o papel indutor da Política Industrial, Tecnológica e de Comércio Exterior (PITCE) e da Política de Desenvolvimento Produtivo (PDP) 
nos setores de Medicamentos, Biotecnologia e Nanotecnologia, como áreas estratégicas e de futuro, por exemplo, para impulsionar o dinamismo econômico, tendo a inovação como pauta preponderante ao crescimento da competitividade do país (DA SILVA et al., 2015). Entre os anos de 2004 a 2013, por intermédio do Ministério da Ciência, Tecnologia e Inovações e Comunicações (MCTIC) e da Financiadora de Estudos e Projetos (FINEP), foi fomentado a título não reembolsável $\mathrm{R} \$ 26.430 .000,00$ (milhões) para estudos envolvendo nanotecnologia com aplicação em fármacos (MCTIC, 2015). Recentemente, o governo também desenvolveu políticas de incentivo à nanotecnologia por meio do Plano Plurianual (PPA) do MCTIC, entre os anos de 2016 a 2019 (MPDG, 2016).

Diante do exposto, este artigo objetiva analisar a dinâmica das atividades de P\&D nacionais em nanotecnologia com aplicação no setor farmacêutico. Tendo em vista os constantes incentivos e políticas governamentais realizadas nas últimas duas décadas, esta análise abrangeu: i) a identificação das linhas de pesquisas criadas; ii) os depósitos de patentes efetuados; iii) os estudos clínicos oriundos das pesquisas e tecnologias brasileiras; iv) as parcerias criadas entre instituições de pesquisa brasileiras e empresas nacionais; e v) os produtos de origem nacional oriundos destas pesquisas. A avaliação conjunta desses resultados permitirá uma análise da incorporação da nanotecnologia pela indústria farmacêutica nacional, bem como as perspectivas de mercado dessas tecnologias e inovações desenvolvidas no Brasil.

\section{Metodologia}

O presente trabalho pode ser considerado um estudo exploratório, integrando pesquisa qualitativa na coleta e análise de dados em diferentes bancos de informações. A pesquisa exploratória visa à descoberta, ao achado, à elucidação de fenômenos ou à explicação daqueles que não eram aceitos apesar de evidentes. A exploração representa, atualmente, um importante diferencial competitivo em termos de concorrência (GONÇALVES, 2014). A pesquisa, neste estudo, foi efetuada conforme Cervo, Bervian e Silva (2006), que estabelecem critérios, métodos e técnicas para sua elaboração, visando oferecer informações sobre o seu objeto e orientar a formulação de hipóteses. A metodologia para busca de linhas de pesquisas objetivou apenas as linhas de pesquisa vinculadas aos grupos de pesquisas do $\mathrm{CNPq}$, dos quais se originam projetos de pesquisa e, por conseguinte, derivam-se os artigos científicos. A busca dos depósitos de patentes foi realizada com enfoque nos fármacos que fazem uso de nanotecnologia e tem aplicação na saúde humana. Para tanto, foi realizado um filtro por meio da leitura manual de cada depósito analisado, obtendo-se aqueles que atenderam ao escopo da pesquisa. Com a obtenção das tecnologias depositadas com origem de pesquisa brasileira, foram selecionadas aquelas que resultaram em ensaios clínicos. A partir destas, efetuou-se a buscas das que resultaram em produtos.

\subsection{Linhas de Pesquisa}

As linhas de pesquisa foram avaliadas com base no Diretório de Grupos de Pesquisa do CNPq (http://lattes.cnpq.br/web/dgp) por meio da busca parametrizada: 1) Consulta na Base Corrente; 2) Termo de busca "nanotecnologia", marcando "todas as palavras", e consultando apenas as "linhas de pesquisa". Os dados foram coletados e as informações relativas às datas 
de criação, de origem, de aplicações terapêuticas e de subdivisões tecnológicas das linhas de pesquisa foram tabuladas. Só foram consideradas válidas ao estudo as linhas de pesquisa envolvendo nanotecnologia e com aplicação ao desenvolvimento de medicamentos.

\subsection{Depósitos de Patentes}

A pesquisa foi realizada utilizando o sistema Questel Orbit Educacional, por meio de convênio do Mestrado Profissional em Propriedade Intelectual e Transferência de Tecnologia para a Inovação. Foram aplicados filtros por meio da utilização de códigos da Classificação Internacional de Patentes (IPC, na sigla em inglês): (i) A61K (preparações para finalidades médicas, odontológicas ou higiênicas na área da saúde) e (ii) B82* (aplicações com uso da nanotecnologia), avaliando-se apenas depósitos no Brasil. O código A61K engloba uma ampla gama de preparações aplicadas na área da saúde. Considerando que a presente pesquisa buscou obter apenas os resultados de depósitos de patentes empregando nanotecnologia com aplicação ao desenvolvimento de medicamentos para uso humano, foi realizada a leitura manual de cada depósito obtido, após a busca supracitada. Os depósitos foram classificados por: 1) data de depósito; 2) tipologia dos depositantes: origem brasileira e estrangeira, realizados por: a) instituições de pesquisa; b) por empresas ou "pessoas físicas" de direito privado; e c) mistas "parcerias entre empresas e instituições de pesquisa"; 3) origem dos depositantes brasileiros de forma individualizada, entre: a) instituições, b) por empresas ou "pessoas físicas" de direito privado; 4) classificação dos depósitos por aplicações terapêuticas, de acordo com o preconizado pela Agência Nacional de Vigilância Sanitária (ANVISA).

\subsection{Estudos Clínicos e Produtos}

A busca dos ensaios clínicos relacionados aos depósitos de patentes encontrados foi realizada por: 1) Busca de ensaios clínicos nos sites das instituições de pesquisa, das empresas brasileiras e em sítios eletrônicos nacionais de divulgação por meio da utilização de informações como descrição do principio ativo, título e número do depósito de patente; 2) Consulta dos ensaios clínicos autorizados e divulgados no site da ANVISA (2018) por meio do título do protocolo após busca com i) palavra nanotecnologia e ii) nome do princípio ativo constante nos depósitos de patentes; 3) Consulta dos ensaios clínicos autorizados e divulgados no site Clinical Trials (2018), fazendo-se a busca com: i) a palavra "nanotecnologia" colocando-se o país "Brasil"; $e$ ii) princípio ativo constante nos depósitos de patentes.

O levantamento dos produtos, oriundos das tecnologias relacionadas aos depósitos de patentes de origem brasileira, foi feito por meio de busca em sites de empresas e instituições de pesquisa que participaram de parcerias com empresas nacionais, e sítios eletrônicos nacionais de divulgação de informações. Ainda, foi consultada a base de dados de produtos do sítio eletrônico STATNANO (https://product.statnano.com/), um banco de dados internacional de produtos nanotecnológicos com uma ampla gama de aplicações industriais. Nesse sítio eletrônico, realizou-se a pesquisa com os seguintes parâmetros de busca: a) pelo país, Brasil; b) por medicamentos para uso em humanos. 


\section{Resultados e Discussão}

A Figura 1 demonstra a evolução temporal da criação das linhas de pesquisa, conforme mostra o site do CNPq: com menos de um ano até quatro anos acumulado em 2018; com cinco a nove anos acumulado em 2013; com 10 a 14 anos acumulado em 2008; e com mais de 15 anos de criação acumulado em 2003. No período entre 2003 até 2018, percebe-se uma evolução crescente no número de linhas de pesquisa vigentes, passando de 40 linhas de pesquisas criadas até 2003, para 152 linhas de pesquisa criadas até 30 de agosto de 2018.

Figura 1 - Evolução temporal da criação dos grupos de pesquisa com os respectivos números de linhas de pesquisa vinculadas no Brasil

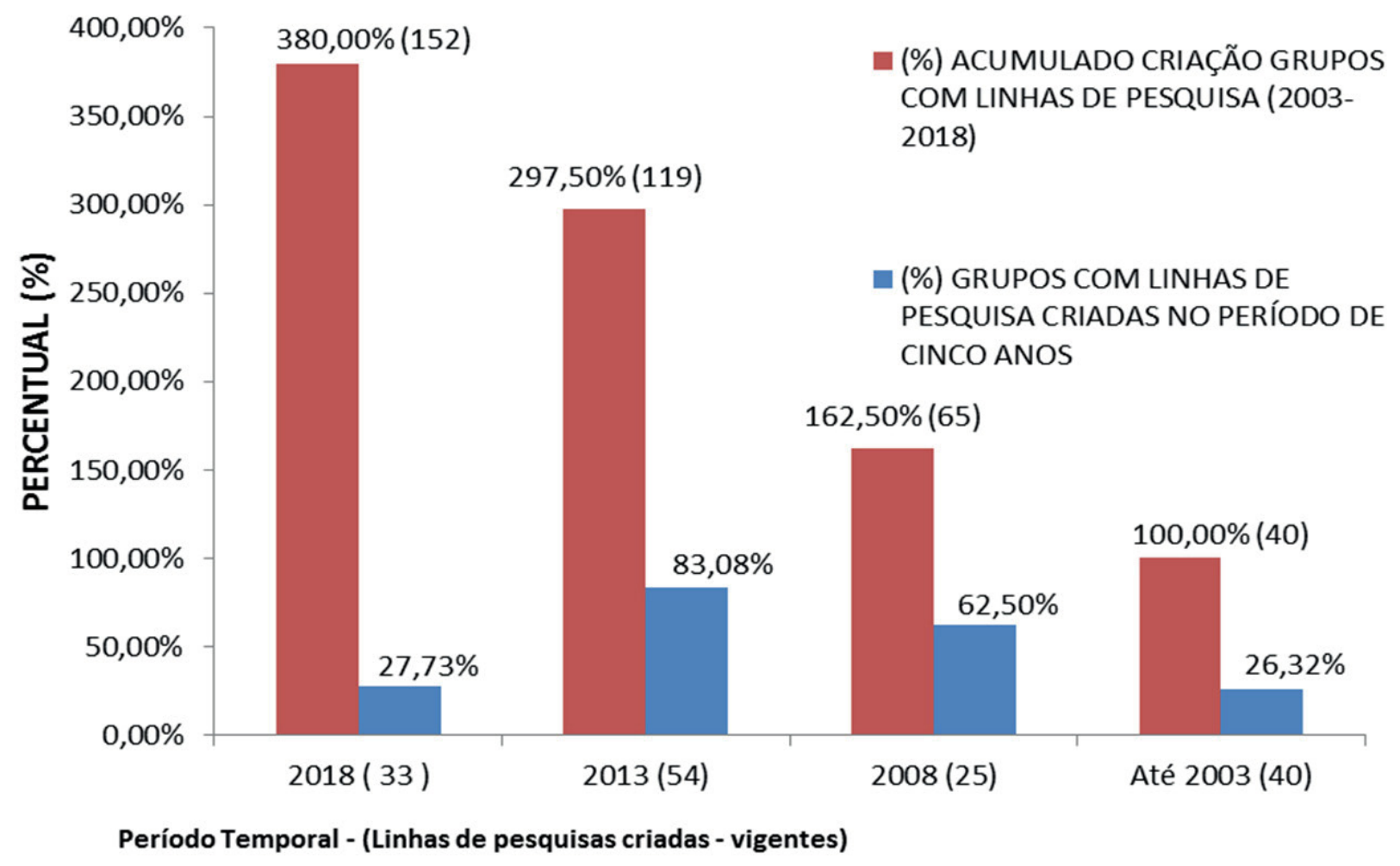

Fonte: Elaborada pelos autores deste artigo (2018)

Por meio do levantamento das linhas de pesquisa, esses dados foram distribuídos por instituições vinculadas, obtendo os detentores de linhas de pesquisa no Brasil, conforme demonstrado no Quadro 1. Pode-se observar que são 54 instituições de pesquisa brasileiras com linhas de pesquisa vigentes. Entre as instituições, destaca-se a Universidade Federal de Pernambuco (UFPE), que aparece em primeiro lugar com 11,18\% (17 linhas de pesquisa), seguida pela Universidade do Estado de São Paulo (UNESP), com 7,89\% (12 linhas de pesquisa), e pela Fundação Oswaldo Cruz, com 5,92\% (nove linhas de pesquisa). 
Quadro 1 - Instituição de origem das linhas brasileiras de pesquisa

\begin{tabular}{|c|c|c|c|c|c|c|c|c|}
\hline INsTITUIÇ:Á & \begin{tabular}{|l|}
$N^{*}$ LINHAS \\
PE SQUTSAS
\end{tabular} & \begin{tabular}{|l|} 
PROPORCA.AO \\
DO TOTAL $(\%)$
\end{tabular} & INSTITUIÇÃo & $\begin{array}{|ll|}N^{*} & \text { LINHAS } \\
\text { PESQUISAS }\end{array}$ & $\begin{array}{l}\text { PROPORCAOAO } \\
\text { DO TOTAL (\%) }\end{array}$ & 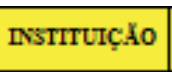 & $\begin{array}{|lr|}N^{*} & \text { LINHAS } \\
\text { PESQUISAS } & \\
\end{array}$ & $\begin{array}{l}\text { FR OPORCYO } \\
\text { DOTOTAL (\%) }\end{array}$ \\
\hline UFPE & 17 & $11,18 \%$ & USP & 3 & $1,97 \%$ & UPE & 1 & $0,66 \%$ \\
\hline UNESP & 12 & $7,89 \%$ & UFRGS & 3 & $1,97 \%$ & UFAC & 1 & $0,66 \%$ \\
\hline FIOCRUZ & 9 & $5,92 \%$ & UFMG & 3 & $1,97 \%$ & UFU & 1 & $0,66 \%$ \\
\hline UFRJ & 7 & $4,61 \%$ & UEPB & 2 & $1,32 \%$ & UFVJM & 1 & $0,66 \%$ \\
\hline IFCE & 6 & $3,95 \%$ & UNIFAP & 2 & $1,32 \%$ & UFSCAR & 1 & $0,66 \%$ \\
\hline $\mathrm{UNB}$ & 5 & $3,29 \%$ & PUCRS & 2 & $1,32 \%$ & CEFET-MG & 1 & $0,66 \%$ \\
\hline UFSC & 5 & $3,29 \%$ & UNVAP & 2 & $1,32 \%$ & UFSC & 1 & $0,66 \%$ \\
\hline IFRJ & 5 & $3,29 \%$ & INST BUTANIAN & 2 & $1,32 \%$ & UFF & 1 & $0,66 \%$ \\
\hline UNCAAMP & 4 & $2,63 \%$ & UNIFAL-MG & 2 & $1,32 \%$ & UNVASF & 1 & $0,66 \%$ \\
\hline UFPB & 4 & $2,63 \%$ & UNCCENTRO & 2 & $1,32 \%$ & UFPA & 1 & $0,66 \%$ \\
\hline UFMS & 4 & $2,63 \%$ & UFBA & 2 & $1,32 \%$ & EMBRAPA & 1 & $0,66 \%$ \\
\hline UEM & 4 & $2,63 \%$ & UNFRA & 1 & $0,66 \%$ & UNIAN & 1 & $0,66 \%$ \\
\hline UFPR & 4 & $2,63 \%$ & UFRR & 1 & $0,66 \%$ & PUC-PR & 1 & $0,66 \%$ \\
\hline UFIF & 4 & $2,63 \%$ & UFPI & 1 & $0,66 \%$ & UFG & 1 & $0,66 \%$ \\
\hline UFAL & 3 & $1,97 \%$ & UNCOESTE & 1 & $0,66 \%$ & UNVAL & 1 & $0,66 \%$ \\
\hline UFSM & 3 & $1,97 \%$ & IF - GOLANO & 1 & $0,66 \%$ & UFRN & 1 & $0,66 \%$ \\
\hline UFAM & 3 & $1,97 \%$ & UTFPR & 1 & $0,66 \%$ & unC-SC & 1 & $0,66 \%$ \\
\hline MAKENZI - SP & 3 & $1,97 \%$ & INAIETRO-BIOTAM & 1 & $0,66 \%$ & UNIARA & 1 & $0,66 \%$ \\
\hline \multicolumn{4}{|c|}{ TOTAL LINHAS DE PE SQUISAS = } & \multicolumn{2}{|c|}{152} & \multicolumn{3}{|c|}{$100,00 \%$} \\
\hline
\end{tabular}

Fonte: Elaborado pelos autores deste artigo (2018)

Posteriormente, foram levantadas as principais aplicações terapêuticas das linhas de pesquisa por subclasse tecnológica. Para tanto, foi utilizada a classificação terapêutica adotada pela ANVISA. Com isso, avaliou-se cada documento da linha de pesquisa e que foram classificados de acordo com as respectivas aplicações terapêuticas descritas, realizando-se o cruzamento entre as linhas de pesquisa, instituições de pesquisa e as aplicações terapêuticas, conforme organizado na Figura 2. Entre as aplicações terapêuticas com maior número de linhas de pesquisa estão os "Agentes Antineoplásicos/Câncer (L-C)" e os "Imunomoduladores contra vírus e bactérias (L-I)" com 25 linhas de pesquisas, ou 11,90\%, cada. Elas possuem, respectivamente, 19 e 13 instituições com linhas de pesquisa. De fato, essas são as aplicações terapêuticas que possuem mais instituições com linhas de pesquisa em desenvolvimento. 
Figura 2 - Linhas de pesquisa por instituição de origem, separadas de acordo com as respectivas aplicações terapêuticas e subdivisões nanotecnológicas

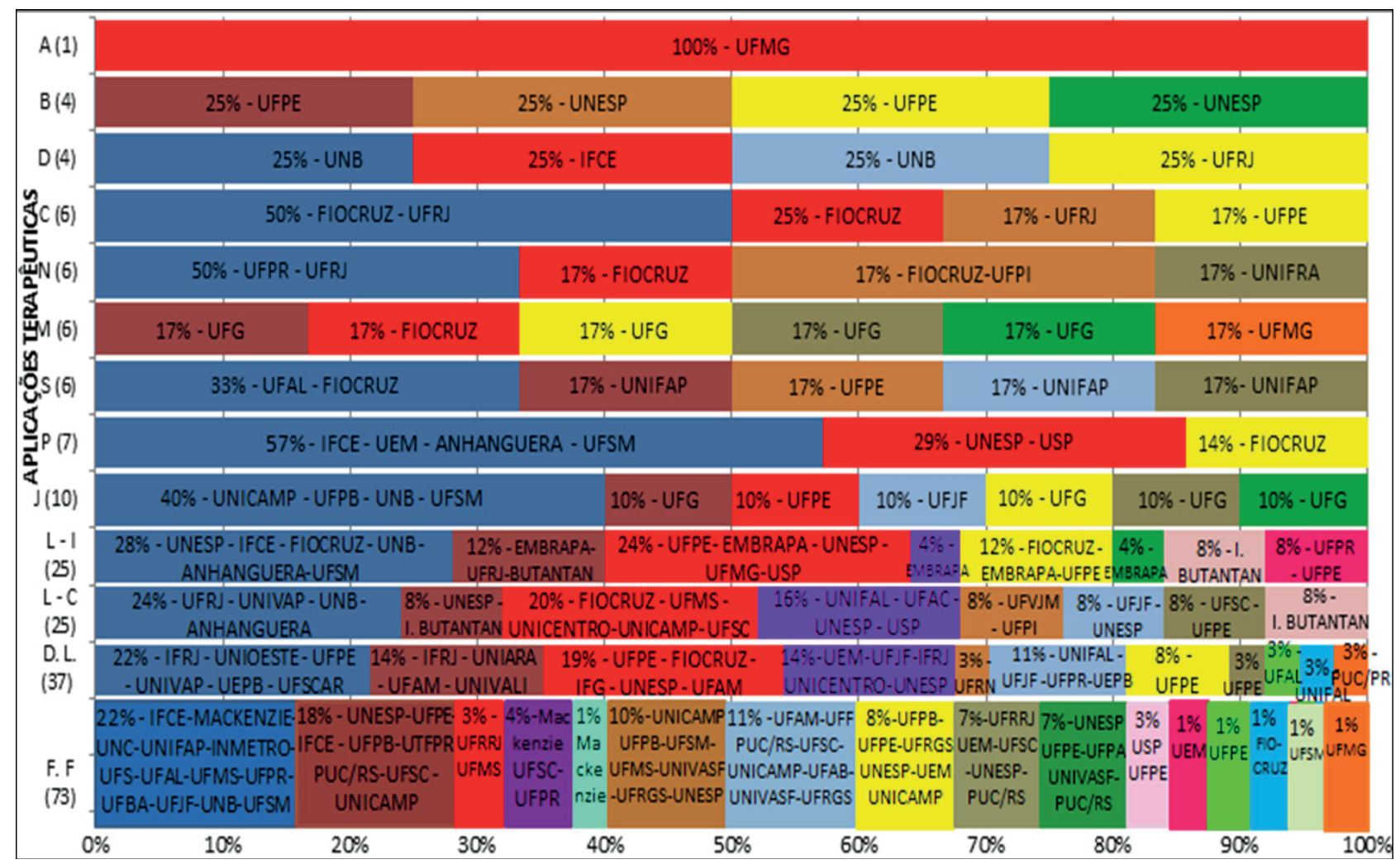

Legenda Figura 2 - Subdivisões Tecnológicas da nanotecnologia: Nanotecnologia Outros; _ Nanosistemas; — Nanopeptídeos; — Nanopolímeros;

Nanometais; Nanocristais; Nanocarreadores/Nanoesferas; Nanofibras; Nanosuspensões; Nanolipossomos; Nanotubos; Nanoproteínas;

Nanoencapsulamento; Nanolipídios; Nanominerais. Classificações de aplicações terapêuticas pela Figura 3 segundo a ANVISA: (A) Aparelho Digestivo e Metabolismo; (B) Sangue e Órgãos Formadores de Sangue; (C) Sistema Cardiovascular; (D) Dermatologia; (D-L) Direcionamento e Liberação de Fármacos; (F-F) Formulação de Fármacos; (G) Sistema Geniturinário e Hormônios Sexuais; (H) Preparações Hormonais; (J) Anti-infecciosos em Geral; (K) Soluções hospitalares; (L) Agentes Antineoplásicos e Imunomoduladores; (M) Sistema Musculoesquelético; (N) Sistema Nervoso Central; (P) Parasitologia; (R) Aparelho Respiratório; (S) Órgãos Sensoriais; (T) Agentes Diagnósticos; (V) Diversos. A aplicação terapêutica segundo a ANVISA (L) - Agentes Antineoplásicos e Imunomoduladores foram subdivididas em: L - C (Agentes Antineoplásicos - Câncer) e L - I (Imunomoduladores que são vacinas contra vírus e bactérias).

Fonte: Elaborada pelos autores deste artigo (2018)

Avaliando-se os depósitos de patentes realizados com os códigos IPC A61K e B82*, foram obtidos 622 depósitos de patentes, no entanto, para esta pesquisa foram aplicáveis $81,19 \%$, ou 505 depósitos, após a leitura de cada depósito realizado no Brasil. Os depósitos realizados no Brasil foram separados pela evolução temporal, no período de publicação entre 1987 e 2018, obtendo-se os depósitos relacionados ao Brasil e ao exterior (Figura 3). Houve uma evolução significativa nos depósitos de origem brasileira, no período entre 2008 até 2016, o que demonstra que essa tecnologia está atingindo sua maturidade em nível nacional. Nota-se, ainda, que o primeiro depósito realizado ocorreu em 2001. 
Figura 3 - Depósitos de patentes realizados por brasileiros e estrangeiros

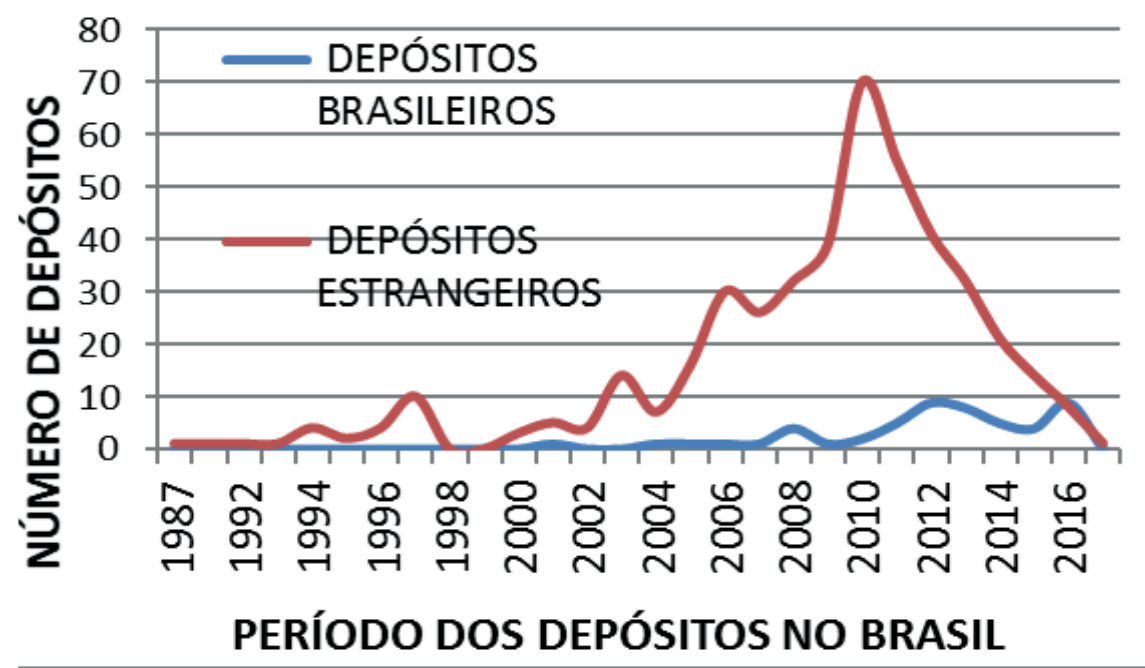

Fonte: Elaborada pelos autores deste artigo (2018)

Posteriormente, foi realizada uma comparação, em termos totais, entre os depositários brasileiros em relação aos depositários do estrangeiro, segmentando-se por tipologias de depositantes: instituições de pesquisa, empresas e mistas (parcerias entre empresas e instituições de pesquisas), conforme a Figura 4. Nesse sentido, observa-se que 10,50\% do total de depósitos são de origem nacional, enquanto $89,50 \%$ são de outros países. Com relação aos depósitos nacionais, observa-se que $81,13 \%$ são produzidos por instituições de pesquisas brasileiras, sendo apenas 7,55\% produzidos por empresas nacionais. Com relação aos depósitos do exterior, $13,27 \%$ são produzidos por instituições de pesquisas e $77,88 \%$ são produzidos por empresas.

Figura 4 - Gráfico absoluto de depositantes no Brasil por tipologia

\section{GRÁFICO ABSOLUTO DE DEPOSITANTES COM PRIORIDADE NO BRASIL}

MISTAS em relação ao total produzidas NO:

(BRASIL / ESTRANGEIRO)

MISTAS em relação ao total depositadas no

BRASIL

EMPRESASem relação ao total produzidas

NO: (BRASIL / ESTRANGEIRO)

EMPRESAS em relação ao total depositadas no BRASIL

ICTS em relação ao total produzidas NO:

(BRASIL / ESTRANGEIRO)

ICTS em relação ao total depositadas no BRASIL

PERCENTUAL TOTAL DE DEPÓSITOS COM PRIORIDADE NO BRASIL

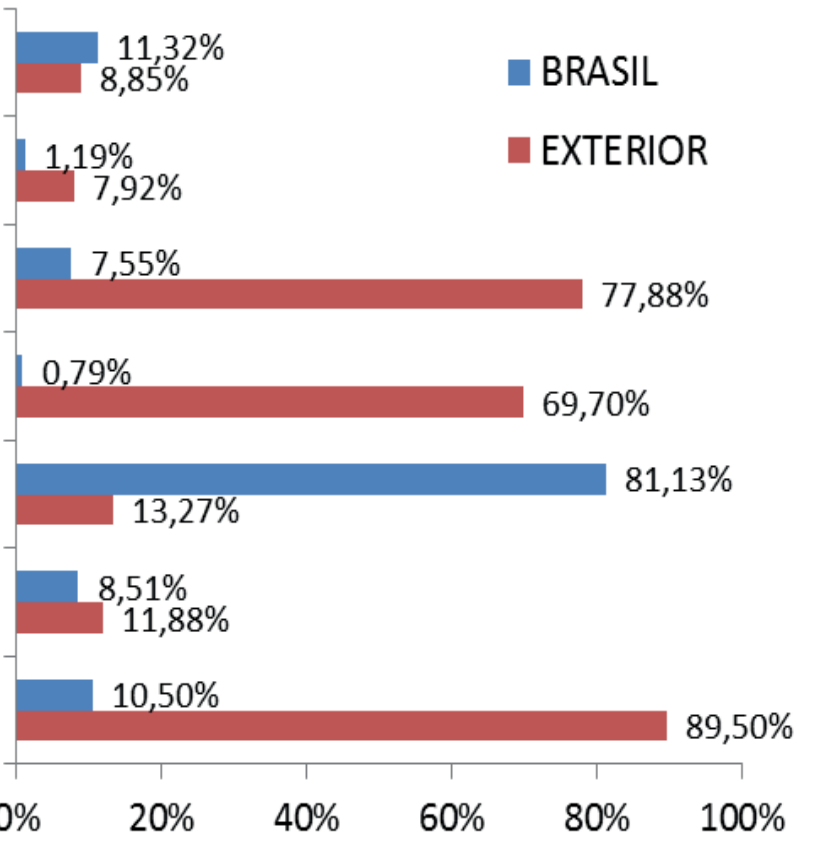

Fonte: Elaborada pelos autores deste artigo (2018) 
Os depósitos de origem brasileira foram, então, separados por instituições depositantes, conforme mostra a Figura 5. Pode-se notar que as instituições de pesquisa UFMG e UNICAMP são as que mais depositaram patentes, sendo que cada instituição contribuiu com 10,29\% do total de depósitos de origem nacional. Entre as empresas nacionais, destaca-se a BIOLAB Sanus com $4,41 \%$ dos depósitos, sendo que todos os seus depósitos foram realizados em parceria com a UFRGS. Com 1,47\% dos depósitos, destacam-se outras cinco empresas: Cristália, Eurofarma, Natura, EMS e Theraskin Farmacêutica.

Figura 5 - Segmentação percentual dos depósitos de patentes de origem brasileira por instituições

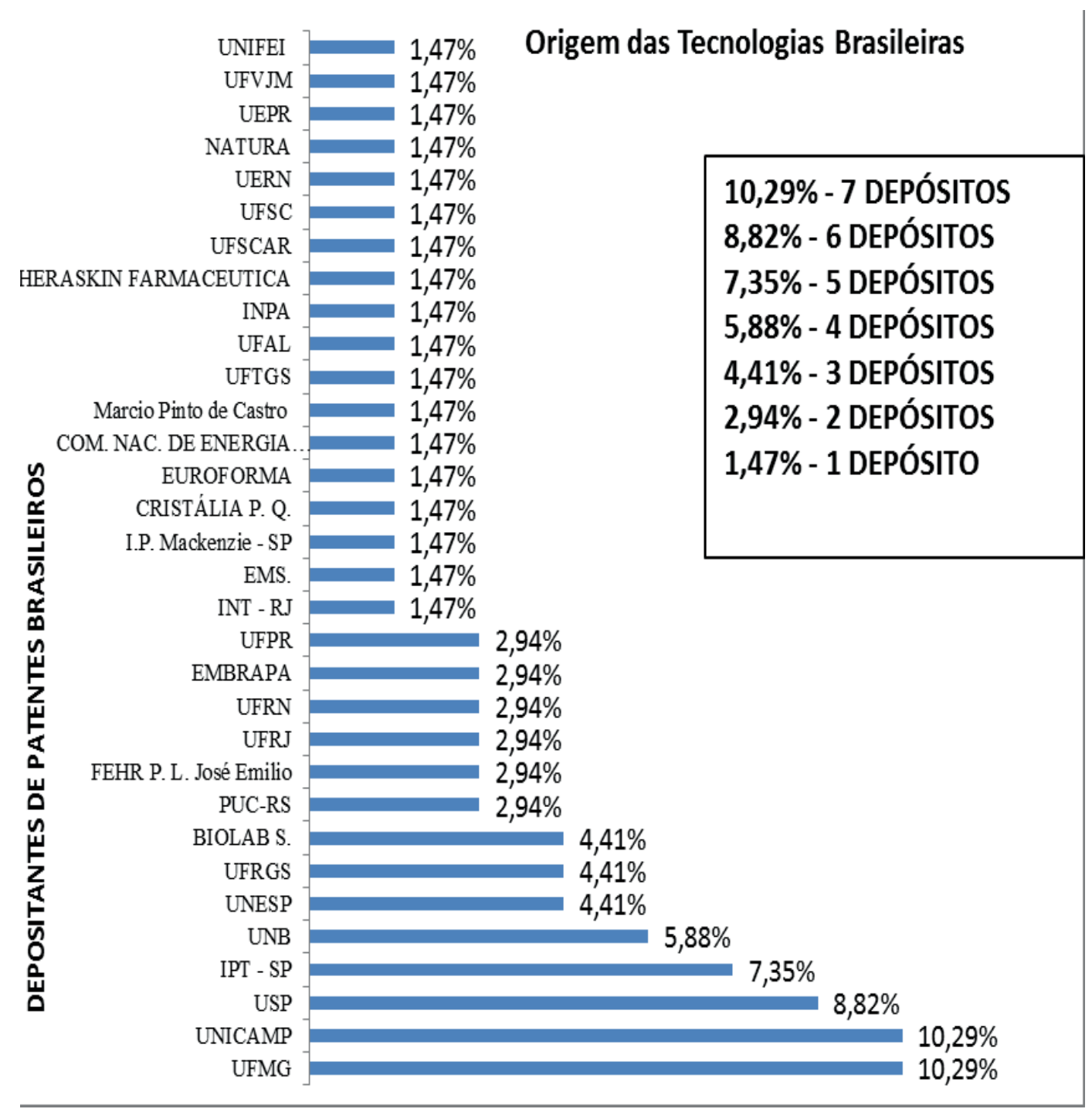

Fonte: Elaborada pelos autores deste artigo (2018)

Ainda, os depósitos de origem nacional foram segmentados de acordo com as aplicações terapêuticas, segundo os preceitos de classificação terapêutica da ANVISA. De acordo com a Figura 6, é possível notar que o "câncer" é a aplicação terapêutica mais utilizada, contando em $25,62 \%$ dos depósitos de origem nacional. É interessante notar que as aplicações terapêuticas com maior porcentagem de patentes (câncer, anti-infecciosos e imunomoduladores) são, também, as aplicações terapêuticas, que mais possuem linhas de pesquisas. Ainda, nota-se que tais aplicações terapêuticas, somadas com as dermatológicas, representam cerca de $61,98 \%$ 
do total de depósitos, quase dois terços de todas as aplicações terapêuticas dos depósitos de origem nacional.

Figura 6 - Aplicações terapêuticas dos depósitos de patentes de origem brasileira

\section{APLICAÇÕES TERAPÊUTICAS DA NANOTECNOLOGIA BRASILEIRA}

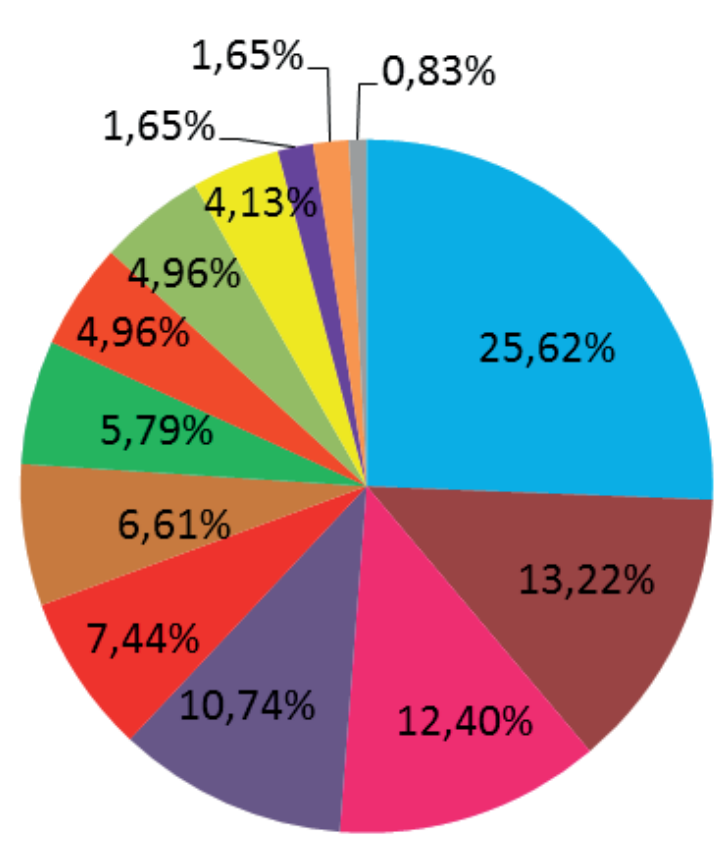

- L - C Câncer

- J - Anti-Infecciosos

D - Dermatologia

- L - Imunomoduladores (VIRUS e Bactérias)

n - S. Nervoso Central

- P - Parasitologia

M - S. Músculo-Esquelético

A - Apar. Digestivo e Metab.

B - Sangue e Órgãos Form. de Sangue

S - Órgãos Sensoriais

- C - S. Cardiovascular

H - Prep. Hormonais

G - S. Geniturinário e Hormônios

Fonte: Elaborada pelos autores deste artigo (2018)

Posteriormente, foi realizada a separação das aplicações terapêuticas dos depósitos de patentes de origem nacional de acordo com as subdivisões tecnológicas para cada instituição/ empresa depositante. De acordo com a Figura 7, a aplicação terapêutica "câncer" apresenta como principal tecnologia empregada os nanopolímeros, com cerca de $30 \%$, seguida pelos nanometais, com 23\%, e os nanocarreadores e nanoesferas, com 16\%. Entre as instituições que depositaram patentes com aplicações ao câncer, destaque para a UFMG e UNICAMP, com quatro diferentes subdivisões tecnológicas, e USP, UFSC, UFRJ e UnB, com três subdivisões tecnológicas. 
Figura 7 - Subdivisões tecnológicas dos depósitos de patentes de origem brasileira segmentados por aplicações terapêuticas

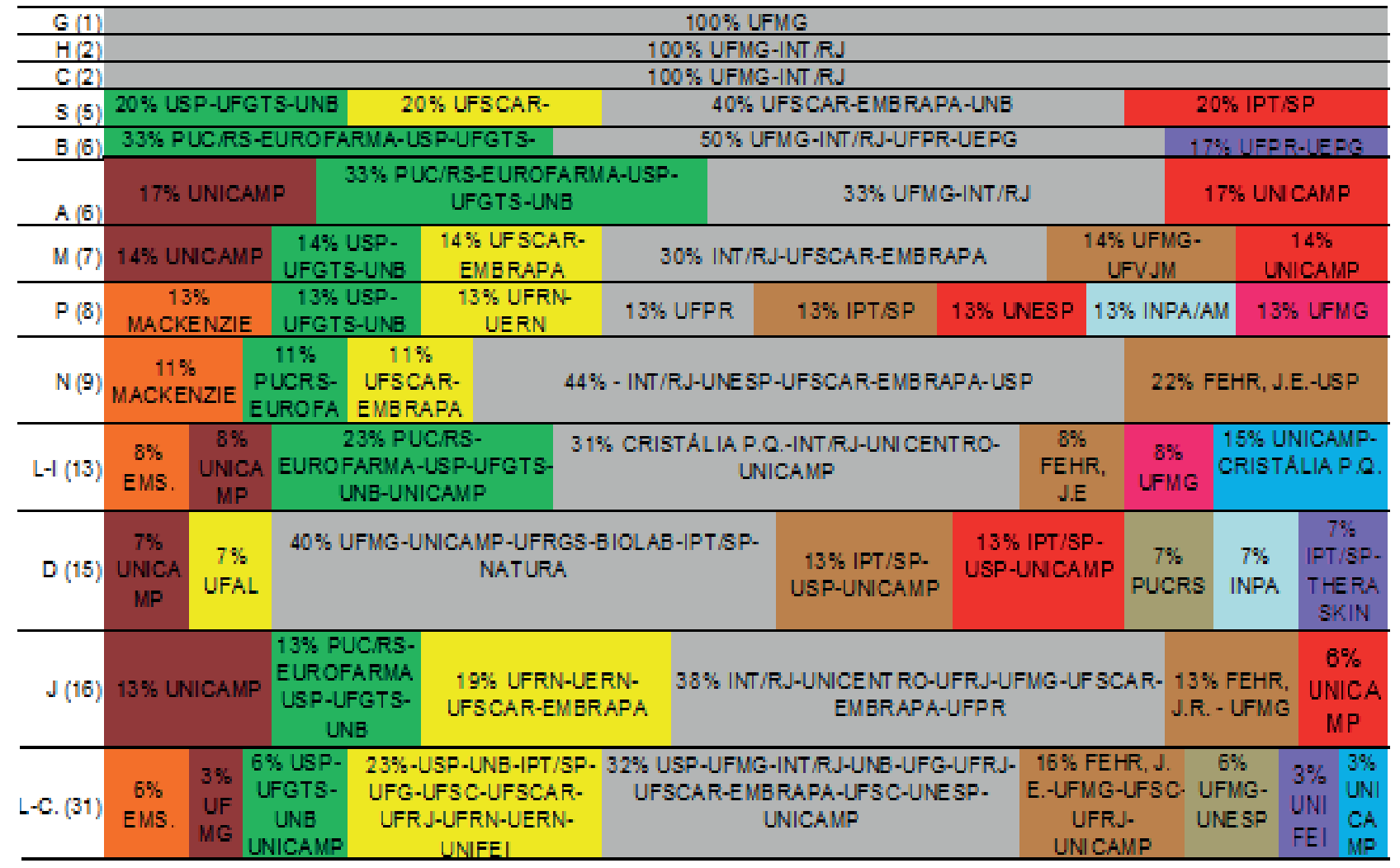

Legenda Figura 7: Nanoemulsões e Nanodispersões; Nanopolímeros; — Nanotubos; Nanohíbridos; Nanocarreadores e Nanoesferas; _ Nanofibras; Nanobastões de Ouro; Nanometais; Nanolipídios; Nanoproteínas; Nanoencapsulamento; Nanominerais de Sílica.

Siglas aplicações terapêuticas pela Figura 8 segundo a ANVISA: (A) Aparelho Digestivo e Metabolismo; (B) Sangue e Órgãos Formadores de Sangue; (C) Sistema Cardiovascular; (D) Dermatologia; (D-L) Direcionamento e Liberação de Fármacos; (F-F) Formulação de Fármacos; (G) Sistema Geniturinário e Hormônios Sexuais; (H) Preparações Hormonais; (J) Anti-infecciosos em Geral; (K) Soluções hospitalares; (L) Agentes Antineoplásicos e Imunomoduladores; (M) Sistema Musculoesquelético; (N) Sistema Nervoso Central; (P) Parasitologia; (R) Aparelho Respiratório; (S) Órgãos Sensoriais; (T) Agentes Diagnósticos; (V) Diversos. A aplicação terapêutica segundo a ANVISA (L) - Agentes Antineoplásicos e Imunomoduladores foram subdivididas em: L - C (Agentes Antineoplásicos - Câncer) e L - I (Imunomoduladores que são vacinas contra vírus e bactérias).

Fonte: Elaborada pelos autores deste artigo (2018)

Na sequência, com base nos depósitos de patentes de origem nacional, foi realizada a busca acerca dos ensaios clínicos, i.e., etapa crítica do desenvolvimento de medicamentos, que foram resultantes de tais depósitos. Assim, o Quadro 2 elenca as instituições/empresas que estão desenvolvendo estudos clínicos a partir de depósitos de patentes encontrados no presente estudo, sendo também ilustrados o princípio ativo, a subdivisão tecnológica e a aplicação terapêutica. De modo geral, percebe-se a importância do envolvimento de instituições de pesquisas públicas brasileiras nas pesquisas com uso da nanotecnologia, as quais vêm resultando no desenvolvimento de novos e inovadores medicamentos no território nacional. Observa-se na relação aos estudos clínicos em andamento que, dos depósitos de patentes de origem nacional previamente descrito, existem seis (6) produtos em desenvolvimento clínico, envolvendo oito (8) Instituições de Pesquisa nacionais e cinco (5) diferentes indústrias farmacêuticas. Destaca-se, contudo, as limitações na busca de ensaios clínicos relacionados aos depósitos de patentes citados neste estudo, ou seja, podem ter ficado de fora outros estudos em andamento e que não estejam cadastrados, divulgados ou registrados. 
Quadro 2 - Ensaios Clínicos realizados envolvendo produtos oriundos de depósitos de patentes de instituições/empresas de origem brasileira

\begin{tabular}{|c|c|c|c|c|c|c|}
\hline $\begin{array}{l}\text { Instituições } \\
\text { de Pesquisas } \\
\text { Brasileiras e/ } \\
\text { ou Empresas }\end{array}$ & $\begin{array}{l}\text { Número do } \\
\text { Depósito de } \\
\text { Patente }\end{array}$ & $\begin{array}{l}\text { Princípio } \\
\text { Ativo }\end{array}$ & $\begin{array}{c}\text { Subdivisão } \\
\text { Tecnológica da } \\
\text { Nanotecnologia }\end{array}$ & $\begin{array}{l}\text { Aplicações } \\
\text { Terapêuticas }\end{array}$ & $\begin{array}{l}\text { Fase do } \\
\text { Ensaio } \\
\text { Clínico }\end{array}$ & $\begin{array}{l}\text { Fonte de } \\
\text { Busca }\end{array}$ \\
\hline \multirow{3}{*}{$\begin{array}{l}\text { BIOLAB- } \\
\text { UFRGS }\end{array}$} & $\begin{array}{l}\text { BR102012022034; } \\
\text { BR102012022036 }\end{array}$ & $\begin{array}{c}\text { Finasterida - } \\
\text { Dapaconazol }\end{array}$ & $\begin{array}{c}\text { Nanopolímeros - } \\
\text { nanoencapsulamento } \\
\text { de fármacos }\end{array}$ & $\begin{array}{c}\text { Calvície; } \\
\text { antimicótico: } \\
\text { fungos e ação } \\
\text { bactericida }\end{array}$ & $\begin{array}{l}\text { FASE } 03 \text { em } \\
\text { andamento }\end{array}$ & $\begin{array}{c}\text { Clinical Trials } \\
\text { (2018) - NCT } \\
\mathbf{0 3 3 2 0 4 8 6}\end{array}$ \\
\hline & \multirow{2}{*}{ BRPI0805854 } & $\begin{array}{l}\text { Lidocaína }+ \\
\text { Prilocaína }\end{array}$ & $\begin{array}{c}\text { Nanopolímeros } \\
\text { nanoencapsulamento } \\
\text { de fármacos }\end{array}$ & Anestésico & $\begin{array}{l}\text { FASE } 03 \\
\text { Concluída }\end{array}$ & $\begin{array}{l}\text { Clinical Trials } \\
\text { (2018) - NCT } \\
\mathbf{0 3 3 6 6 2 4 6}\end{array}$ \\
\hline & & $\begin{array}{l}\text { Lidocaína }+ \\
\text { Prilocaína }\end{array}$ & $\begin{array}{c}\text { Nanopolímeros } \\
\text { nanoencapsulamento } \\
\text { de fármacos }\end{array}$ & Anestésico & $\begin{array}{l}\text { FASE } 01 \\
\text { Concluída }\end{array}$ & $\begin{array}{c}\text { Clinical Trials } \\
\text { (2018) - NCT } \\
\mathbf{0 3 4 4 1 8 4 1}\end{array}$ \\
\hline $\begin{array}{l}\text { IFCE/UFS/ } \\
\text { UNIV. } \\
\text { POTIGUAR - } \\
\text { EVIDENCE }\end{array}$ & BRPI1002486 & $\begin{array}{c}\text { Estriol }(0,1 \%) \\
+ \text { Estradiol } \\
(0,25 \%) e \\
\text { Progesterona }\end{array}$ & $\begin{array}{l}\text { Nanoformulação } \\
\text { transdérmica }\end{array}$ & $\begin{array}{c}\text { Reposição } \\
\text { Hormonal - } \\
\text { Hormonioterapia }\end{array}$ & $\begin{array}{l}\text { FASE } 02 \\
\text { Concluída }\end{array}$ & $\begin{array}{l}\text { Clinical Trials } \\
\text { (2018) - NCT } \\
\mathbf{0 2 0 3 3 5 1 2}\end{array}$ \\
\hline $\begin{array}{l}\text { EUROFARMA } \\
\text { - PUCRS }\end{array}$ & BRPI0705319 & Resveratrol & $\begin{array}{c}\text { Nanoproteínas - } \\
\text { entrega de fármacos }\end{array}$ & $\begin{array}{l}\text { Anti-inflamatório } \\
\text { com potencial } \\
\text { ação em doenças } \\
\text { crônicas como } \\
\text { cardiovasculares, } \\
\text { diabetes, neuro- } \\
\text { degenerativas }\end{array}$ & $\begin{array}{l}\text { FASE } 01 \\
\text { Concluída }\end{array}$ & $\begin{array}{l}\text { ANVISA } \\
\text { (2018): } \\
\text { N. CE: } \\
\text { 146/2012 }\end{array}$ \\
\hline \multirow{3}{*}{$\begin{array}{c}\text { Transferência } \\
\text { UFMG- } \\
\text { UNIÃO } \\
\text { QUÍMICA }\end{array}$} & \multirow{3}{*}{ PI01055097 } & \multirow{3}{*}{$\begin{array}{c}\text { Angiotensina } \\
\text { ANG-(1-7) }\end{array}$} & \multirow{3}{*}{$\begin{array}{l}\text { Nanomicelas - } \\
\text { nanopolímeros }\end{array}$} & $\begin{array}{l}\text { Formulação oral } \\
\text { da Ang-(1-7) para } \\
\text { o tratamento de } \\
\text { cardiomiopatia, } \\
\text { hipertensão } \\
\text { arterial e } \\
\text { síndrome } \\
\text { metabólica. }\end{array}$ & $\begin{array}{l}\text { FASE } 01 \\
\text { Concluída }\end{array}$ & $\begin{array}{l}\text { INCT - } \\
\text { Nanobiofar, } \\
\text { (2018) }\end{array}$ \\
\hline & & & & $\begin{array}{c}\text { Formulação } \\
\text { endovenosa para } \\
\text { tratamento da } \\
\text { pré-eclâmpsia }\end{array}$ & $\begin{array}{l}\text { FASE-0/2b } \\
\text { Concluída }\end{array}$ & $\begin{array}{l}\text { INCT - } \\
\text { Nanobiofar, } \\
\text { (2018) }\end{array}$ \\
\hline & & & & $\begin{array}{c}\text { Formulação tópica } \\
\text { para o tratamento } \\
\text { da alopecia. }\end{array}$ & $\begin{array}{l}\text { (FASE 03) } \\
\text { Concluída }\end{array}$ & $\begin{array}{l}\text { INCT - } \\
\text { Nanobiofar, } \\
\text { (2018) }\end{array}$ \\
\hline $\begin{array}{c}\text { Licença da } \\
\text { Patente da } \\
\text { Cristália para } \\
\text { governo de } \\
\text { SP e Federal - } \\
\text { Pesquisas sendo } \\
\text { realizadas pela } \\
\text { FIOCRUZ } \\
\text { E UNESP }\end{array}$ & PI0503817 & Sílica SBA-15 & $\begin{array}{l}\text { nano-polímeros, } \\
\text { nanotubos de sílica. }\end{array}$ & $\begin{array}{c}\text { Vacina por via } \\
\text { oral (comprimido) } \\
\text { contra a } \\
\text { hepatite } \mathrm{B}\end{array}$ & $\begin{array}{l}\text { Estudos } \\
\text { pré-clínicos } \\
\text { concluídos. } \\
\text { Estudos } \\
\text { clínicos em } \\
\text { andamento }\end{array}$ & $\begin{array}{l}\text { (ABIPTI, } \\
2015)\end{array}$ \\
\hline
\end{tabular}

Fonte: Elaborado pelos autores deste artigo (2018) 
Posteriormente, a busca por produtos oriundos dos depósitos de patentes de origem brasileira foi realizada, conforme previamente descrito. Os dados obtidos no Quadro 3 consideraram os resultados dos depósitos de patentes de origem brasileira, as parcerias realizadas e as transferências feitas entre instituições de pesquisas e empresas. Destaca-se que, em parceria com a UFRGS, a Biolab desenvolveu um nanoanestésico tópico, o Nanorap ${ }^{\circledR}$, com ação ultrarrápida. A mesma empresa, e com a mesma parceira, também está desenvolvendo o antifúngico Zilt@. A empresa Evidence está desenvolvendo o produto Biolipídeo B2®, que utiliza nanotecnologia, propiciando uma liberação de hormônios por via transdérmica para reposição hormonal. O depósito de patente que resultou no produto é fruto da parceria entre a empresa e a Universidade Federal do Ceará (UFC) (CGEE, 2012).

Quadro 3 - Produtos de origem brasileira com nanotecnologia

\begin{tabular}{|c|c|c|c|c|c|c|}
\hline $\begin{array}{c}\text { FORMA } \\
\text { INTERAÇÃO } \\
\text { - INSTITUIÇÕES } \\
\text { DE PESQUISAS } \\
\text { BRASILEIRAS E } \\
\text { EMPRESAS }\end{array}$ & $\begin{array}{c}\text { Depósito de } \\
\text { Patente }\end{array}$ & $\begin{array}{c}\text { SubClasse } \\
\text { TeCnOlógICA }\end{array}$ & Produto & $\begin{array}{l}\text { APLICAÇÕES } \\
\text { TERAPÊUTICAS }\end{array}$ & $\begin{array}{c}\text { Princípio } \\
\text { Ativo }\end{array}$ & $\begin{array}{l}\text { SitUAÇÃo DE } \\
\text { REGISTRO }\end{array}$ \\
\hline BIOLAB-UFRGS & $\begin{array}{l}\text { BR102012022034; } \\
\text { BR102012022036 }\end{array}$ & $\begin{array}{c}\text { nanopolímeros } \\
\text { nanoencapsulamento } \\
\text { de fármacos }\end{array}$ & ZILT® & $\begin{array}{c}\text { calvície; } \\
\text { antimicótico }\end{array}$ & $\begin{array}{l}\text { Finasterida - } \\
\text { Dapaconazol }\end{array}$ & $\begin{array}{c}\text { Fase de registro } \\
\text { na ANVISA }\end{array}$ \\
\hline BIOLAB-UFRGS & BRPI0805854 & $\begin{array}{c}\text { nanopolímeros - } \\
\text { nanoencapsulamento } \\
\text { de fármacos }\end{array}$ & NANORAP $®$ & $\begin{array}{l}\text { anestésico; } \\
\text { analgésico }\end{array}$ & $\begin{array}{l}\text { Prilocaína e } \\
\text { a Lidocaína }\end{array}$ & $\begin{array}{c}\text { Fase de registro } \\
\text { na ANVISA }\end{array}$ \\
\hline $\begin{array}{l}\text { EVIDENCE - } \\
\text { IFCE/UFS/ } \\
\text { UNIV. } \\
\text { POTIGUAR. }\end{array}$ & BRPI1002486 & $\begin{array}{c}\text { nanoformulação } \\
\text { transdérmica } \\
\text { com lipídios }\end{array}$ & $\begin{array}{c}\text { BIOLIPIDEO } \\
\text { B2 } \AA\end{array}$ & $\begin{array}{l}\text { Reposição } \\
\text { hormonal }\end{array}$ & $\begin{array}{l}\text { Estriol }(0,1 \%) \\
+ \text { Estradiol } \\
(0,25 \%) e \\
\text { Progesterona }\end{array}$ & $\begin{array}{c}\text { Dispensa } \\
\text { registro na } \\
\text { ANVISA } \\
\text { - produto } \\
\text { manipulado } \\
\text { aprovado n. } \\
\text { de registro } \\
\text { empresa } \mathbf{0 1 7 3 6 2 3} \\
\text { - classe b2 - } \\
\text { rdc } \mathbf{3 4 4} / \mathbf{1 9 9 8}\end{array}$ \\
\hline
\end{tabular}

Fonte: Elaborado pelos autores deste artigo (2018)

\section{Considerações Finais}

Em conjunto, o presente estudo evidenciou um crescimento de $380 \%$ nas linhas de pesquisas envolvendo nanotecnologia e vinculadas a grupos de pesquisa de instituições brasileiras. No período entre 2003 até 2018, ocorreu uma evolução crescente no número de linhas de pesquisa vigentes, passando de 40 linhas até 2003, para 152 linhas em 30 de agosto de 2018.

Por meio da análise dos depósitos de patentes, oriundas das pesquisas supracitadas, foram observadas 11 diferentes aplicações terapêuticas envolvendo nanotecnologia. Entre os estudos desenvolvidos, percebe-se uma tendência ascendente de linhas de pesquisas voltadas ao desenvolvimento de agentes antineoplásicos e imunomoduladores. Nessa temática, observa-se que a FIOCRUZ, a UNESP e a UFPE apresentaram um número significativo de linhas de pesquisas em andamento. 
Com relação aos depósitos de patentes, com prioridade no Brasil, observa-se que apenas $3,77 \%$ de todos os depósitos brasileiros ocorreram até o ano de 2004 . Entre o período de 2004 até 2012 houve um crescimento no percentual de depósitos, de origem brasileira, de cerca de $32 \%$. A partir de 2013, até o ano de 2017, os depósitos de patentes cresceram na ordem de 50,94\%. De modo notório, houve uma significativa evolução no número de depósitos de patentes de origem nacional. Destaca-se a elevada taxa percentual de depósitos oriundos de instituições brasileiras de pesquisas ( $81,1 \%$ dos depósitos do Brasil). Entre as principais aplicações terapêuticas observadas, destacam-se os agentes antineoplásicos, anti-infecciosos, imunomoduladores e dermatológicos.

Por fim, o presente estudo demonstrou que o Brasil ainda está absorvendo de forma incipiente as tecnologias associadas às patentes de origem nacional. Contudo, ressalta-se que, nessa área temática, a transformação de pesquisas em produtos é naturalmente mais lenta, em virtude da necessidade de complexos estudos pré-clínicos e clínicos. Nesse sentido, salienta-se que mais de dois terços dos depósitos de origem nacional foram realizados após o ano de 2012, de modo que muitas das tecnologias depositadas recentemente possam produzir novos e inovadores medicamentos de origem brasileira nos próximos anos.

\section{Referências}

ABDI - AGÊNCIA BRASILEIRA DE DESENVOLVIMENTO INDUSTRIAL. Relatório

nanotecnologia na área da saúde: mercado, segurança e regulação. Janeiro de 2013. Disponível em: http://www.abdi.com.br/Estudo/relatorio-nanote cnologia.pdf. Acesso em: 9 set. 2017.

ANVISA - AGÊNCIA NACIONAL DE VIGILÂNCIA SANITÁRIA. Consulta de Ensaios Clínicos Autorizados pela Anvisa. 2018. Disponível em: http://www7.anvisa.gov.br/Datavisa/Consulta_ Comunicados/Consulta_CE_Autorizados.asp. Acesso em: 13 jul. 2018.

ALENCAR M. S. M.; ANTUNES A. M. S. Análise de patentes pela classificação em nanotecnologia e sua aplicação em fármacos. In: ANTUNES A. M. S.; MAGALHÃES J. L. Patenteamento e

Prospecção Tecnológica no Setor Farmacêutico. Rio de Janeiro: Interciência, 2008. p. 159-180.

ARONSON, J. K. Something new every day: defining innovation and innovativeness in drug therapy. The Journal of Ambulatory Care Management, [S.1.], v. 31, n. 1, p. 65-68, jan.-mar. 2008. Disponível em: https://journals.lww.com/ambulatorycaremanagement/pages/articleviewer.aspx?year= 2008\&issue $=01000 \&$ article $=00011 \&$ type $=$ abstract. Acesso em: 23 abr. 2018.

ABIPTI - ASSOCIAÇÃO BRASILEIRA DAS INSTITUIÇÕES DE PESQUISA TECNOLÓGICA E INOVAÇÃO. Pesquisa brasileira desenvolve vacina via oral contra a hepatite B. 2015. Disponível em: http://www.agenciacti. com.br/agencia-flat/?p=2356. Acesso em: 25 set. 2018.

BNDES - BANCO NACIONAL DE DESENVOLVIMENTO ECONÔMICO E SOCIAL. Ensaios clínicos no Brasil: competitividade internacional e desafios. Brasília, 2012. Disponível em: https:// web.bndes.gov.br/bib/jspui/bitstr eam/1408/1504/2/A\%20set.36_Ensaios\%20cl\%C3\%ADnicos\%20 no\%20Brasil.pdf. Acesso em: 20 set. 2018.

CAIXETA, A. V.; BINSFELD, P. C. Nanomedicamentos e Nanocarreadores de drogas para o uso terapêutico de Câncer. Goiás: Programa de Pós-Graduação em Vigilância Sanitária, 2013. Disponível em: http://www.cpgls.pucgoias.edu.br/8mostra/Artigos/SAUDE\%20E\%20BIOLOGICAS/ Nanomedicamentos\%20e\%20Nanocarreadores\%20de\%20drogas\%20para\%20o\%20uso\%20 terap\%C3\%AAutico\%20de\%20C\%C3\%A2ncer.pdf. Acesso em: 15 dez. 2017. 
CGEE - CENTRO DE GESTÃO E ESTUDOS ESTRATÉGICOS. II Workshop Nanotecnologias: da ciência ao mundo dos negócios, Relatório Final. Brasília, 2012. Disponível em: https:/www.cgee.org. br/documents/10195/734063/Relat\%C3\%B3rio+Final_formatado_8336.pdf/3d65079b-2c78-4ce5b0d2-5aa2a26d1c03?version=1.0. Acesso em: 12 out. 2018.

CNANO-UFRGS - CENTRO DE NANOCIÊNCIA E NANOTECNOLOGIA DA UNIVERSIDADE FEDERAL DO RIO GRANDE DO SUL. Homepage. [2018]. Disponível em: https://www.ufrgs.br/ cnano/. Acesso em: 10 set. 2018.

CLINICAL TRIALS. Ensaios Clínicos. 2018. Disponível em: https://clinicaltrials.gov/. Acesso em: 25 set. 2018.

CNPQ - CONSELHO NACIONAL DE DESENVOLVIMENTO CIÊNTIFICO E TECNOLÓGICO.

Linhas de pesquisa. 2018. Disponivel em: http://lattes.cnpq.br/web/dgp/glossario ;jsessionid $=4 G J 7 s q Z j P J w S o R g U 4 B j U 0 r Y q . u n d e f i n e d ? p \_p \_i d=54$ INSTANCE_QoMcDQ9EVoSc\&p_p_ lifecycle $=0 \& p \_p \_s t a t e=$ normal\&p_p_mode $=$ view\&p_p_col_id $=$ column-3\&p_p_col_ count $=1 \&$ 54_INSTANCE_QoMcDQ9EVoSc_struts_action=\%2Fwiki_display\%2Fview\&_54_ INSTANCE_QoMcDQ9EVoSc_nodeName=Main\&_54_INSTANCE_QoMcDQ9EVoSc_ title $=$ Linhas + de + pesquisa. Acesso em: 9 ago. 2018.

DA SILVA, K. et al. Domínios Tecnológicos das Patentes Acadêmicas no Brasil: Interação dos Inventores Acadêmicos nos Pedidos de Patentespela Via Pct 2002-2012, 2015. In: CONGRESSO LATINO-IBEROAMERICANO DE GESTÃO DA TECNOLOGIA, 16., 2015, Porto Alegre. Anais [...] Porto Alegre: ALTEC, 2015. Disponível em: http://altec2015.nitec.co/altec/papers/913.pdf. Acesso em: 10 maio 2018.

DIMER, F. A. et al. Impactos da Nanotecnologia na Saúde: Produção de Medicamentos. Química Nova, [S.l.], v. 36, n. 10, 2013. Disponível em: http://quimicanova.sbq.org.br/detalhe_artigo. asp?id=3046. Acesso em: 11 dez. 2017.

EUROFARMA. Eurofarma, em parceria com PUCRS, licencia patentes para desenvolver medicamento inovador à base de resveratrol. [2008]. Disponível em: https:/www.eurofarma. com.br/release/eurofarma-em-parceria-com-pucrs-licencia-patentes-para-desenvolver-medicamentoinovador-a-base-de-resveratrol/. Acesso em: 15 set. 2018.

EVERS, P. Nanotechnology in Medical Applications: The Global Market. BccResearch, [S.I.], September 2015. Disponível em: https://www.bccresearch.com/market-research/healthcare/ nanotechnology-medical-applications-market-hlc069c.html. Acesso em: 7 set. 2017.

EVIDENCE. Dossiê Científico Biolipideo. 2012. Disponível em: https://issuu.com/grupoevidence4/ docs/dossie_biolip_deo_b2_. Acesso em: 10 out. 2018.

FAIYAZUDDIN, U. A. Md. Patented Nanopharmaceuticals: A Hope for Patent Expired Formulations. OMICS Internacional Intel Prop Rights, [S.I.], v. 2, n. 2, p. e104, fev. 2014. ISSN: 2375-4516. Disponível em: https:/www.omicsonline.org/open-access/patented-nanopharmaceuticals-a-hope-forpatent-expired-formulations-ipr.1000e104.php?aid=23933. Acesso em: 7 set. 2017. DOI: https://doi. org/10.4172/2375-4516.1000e104 .

GOMES, R. et al. O novo cenário de concorrência na indústria farmacêutica brasileira. Complexo Industrial da Saúde. BNDES Setorial, Rio de Janeiro, v. 39, p. 97-134, 2014. Disponível em: https://web.bndes.gov.br/bib/jspui/handle/1408/4541. Acesso em: 24 out. 2018.

GONÇALVES, H. A. Manual de metodologia da pesquisa cientifica. 2. ed. São Paulo: Avercamp, 2014. 
INCT - INSTITUTOS NACIONAIS DE CIÊNCIA E TECNOLOGIA. Homepage. 2018. Disponível em: http://inct.cnpq.br/web/inct-nanobiofar/resultados/. Acesso em: 4 dez. 2018.

KAITIN, K. I. Deconstructing the Drug Development Process: the New Face of Innovation. Clin Pharmacol Ther, [S.l.], v. 87, n. 3, p. 356-361, feb. 2010. Disponível em: https://www.ncbi.nlm.nih. gov/pmc/articles/PMC2953249/. Acesso em: 10 abr. 2018.

LIU, C.; CONSTANTINIDES, P. C.; LI, Y. Research and development in drug innovation: reflections from the 2013 bioeconomy conference in China, lessons learned and future perspectives. Acta Pharm Sin B, [S.l.], v. 4, n. 2, p. 112-199, 18 feb. 2014. Disponível em: https://www.ncbi.nlm.nih. gov/pmc/articles/PMC4590300/. Acesso em: 28 nov. 2018.

MARTINS, D. Brasil realiza poucos testes clínicos de medicamentos em humanos.

Folha de São Paulo, São Paulo, 2017. Disponível em: https:/www1.folha.uol.com.br/ seminariosfolha/2017/04/1878809-brasil-realiza-poucos-testes-clinicos-de-medicamentos-emhumanos.shtml. Acesso em: 5 out. 2018.

MCTIC - MINISTÉRIO DA CIÊNCIA, TECNOLOGIA E INOVAÇÃO. Ações e Programas PPA 2012-2015. [2015]. Disponível em: http://www.mctic.gov.br/mctic/export/sites/institucional/arquivos/ Programas_Acoes_e_Unidades_Responsaveis.xls. Acesso em: 9 set. 2017.

MPDG - MINISTÉRIO DO PLANEJAMENTO, DESENVOLVIMENTO E GESTÃO. Relatório Anual de Avaliação 2016-2019. [2016]. Disponível em: http://www.planejamento.gov.br/secretarias/ upload/arquivo/spi-1/ppa-2016-2019/rel_anual_de_avaliacao_ppa_2016_2019_volume_i.pdf. Acesso em: 9 set. 2017.

MIGUEL, S. Pesquisa em humanos no Brasil poderá avançar com nova legislação. Instituto de Estudos Avançados da Universidade de São Paulo (IEA), 2016. Disponível em: http://www.iea. usp.br/noticias/pesquisa-em-humanos-no-brasil-podera-avancar-com-nova-legislacao. Acesso em: 9 out. 2018.

PFEIFER, M. O. A vez das moléculas hightech. Interfarma, [S.I.], 2015. Disponível em: https://www. interfarma.org.br/noticias/738. Acesso em: 31 set. 2018.

PORTAL DE NANOTECNOLOGIA BRASILEIRO (NANOCOM). Incrementa lança primeiro fármaco brasileiro desenvolvido com nanotecnologia. 2007. Disponível em: http://nanocom. com.br/inovacao/incrementa-lanca-primeiro-far maco-brasileiro-desenvolvido-com-nanotecnologia/. Acesso em: 8 ago. 2018.

SANT'ANNA, L. S.; ALENCAR, M. S. M.; FERREIRA, A. P. Patenteamento em Nanotecnologia no Brasil: desenvolvimento, potencialidades e reflexões para o meio ambiente e a saúde humana. Química Nova, São Paulo, v. 36, n. 2, p. 348-353, 2013. Disponível em: http://www.scielo.br/scielo. php?script $=$ sci_arttext\&pid=S0100-40422013000200024. Acesso em: 2 set. 2017.

STATNANO. Informações e estatísticas sobre ciência, tecnologia e indústria baseadas em nanotecnologia. 2018. Disponível em: http://statnano.com. Acesso em: 10 set. 2018.

VASCONCELOS, Y. Inovação em fármacos. Revista FAPESP, [S.l.], Edição 238, dez. 2015. Disponível em: http://revista pesquisa.fapesp.br/2015/12/15/moleculas-inovadoras/?cat=tecnologia. Acesso em: 21 set. 2018.

VASCONCELOS, Y. Inovação em fármacos. Revista FAPESP, [S.l.], Edição 260, out. 2017. Disponível em: http://revistapesquisa.fapesp.br/2017/10/25/inovacao-em-farmacos/. Acesso em: 21 set. 2018. 


\section{Sobre os Autores}

\section{Tiago Giuriatti}

E-mail: tiagogideal@hotmail.com

Mestrado Profissional em Propriedade Intelectual e Transferência de Tecnologia para Inovação (2019) pela UFSC.

Endereço profissional: Instituto Federal Catarinense, Blumenau, SC. CEP: 89051-000.

\section{Manuel Adalberto Alfaro De Prá}

E-mail: manueldepra78@gmail.com

Doutor em Biotecnologia e Biociências (2017) pela UFSC.

Endereço profissional: Entourage Phytolab, Valinhos, SP. CEP: 13274-465.

\section{Fernando Richartz}

E-mail: Fernando.richartz@ufsc.br Doutor em Contabilidade (2016) pela UFSC.

Endereço profissional: Departamento de Ciências Contábeis, Centro Socioeconômico, Universidade Federal de Santa Catarina, Florianópolis, SC. CEP: 88040-900.

\section{Eduardo Luiz Gasnhar Moreira}

E-mail:eduardo.luiz@ufsc.br Doutor em Neurociências (2013) pela UFSC.

Endereço profissional: Departamento de Ciências Fisiológicas, Centro de Ciências Biológicas, Universidade Federal de Santa Catarina, Florianópolis, SC. CEP: 88040-900. 